\title{
molecules
}

ISSN 1420-3049

www.mdpi.com/journal/molecules

Article

\section{Synthesis, Structure and Insecticidal Activities of Some Novel Amides Containing $N$-Pyridylpyrazole Moeities}

\author{
Wei-Li Dong ${ }^{1, *}$, Jing-Ying $\mathrm{Xu}^{2}$, Li-Xia Xiong ${ }^{2}$ and Zheng-Ming $\mathrm{Li}^{2}$
}

1 Tianjin Key Laboratory on Technologies Enabling Development of Clinical Therapeutics and Diagnostics (Theranostics), School of Pharmacy, Tianjin Medical University, Tianjin 300070, China

2 State Key Laboratory of Elemento-Organic Chemistry, National Pesticide Engineering Research Center, Nankai University, Tianjin 300071, China; E-Mails: xujunying@mail.nankai.edu.cn (J.-Y.X.); xionglixia@nankai.edu.cn (L.-X.X.); zml@nankai.edu.cn (Z.-M.L.)

* Author to whom correspondence should be addressed; E-Mail: wldong33@163.com; Tel./Fax: +86-22-2354-2014.

Received: 1 August 2012; in revised form: 13 August 2012 / Accepted: 18 August 2012 / Published: 31 August 2012

\begin{abstract}
In our search for environmentally benign insecticides with high activity, low toxicity and low residue, a novel series of amides containing $N$-pyridylpyrazole moieties were designed and synthesized. The structures of the title compounds were characterized and confirmed by ${ }^{1} \mathrm{H}-\mathrm{NMR}$ and elemental analysis. Furthermore, the structure of compound $\mathbf{7 l}$ was determined by single crystal X-ray diffraction. The preliminary bioassay tests showed that some of them exhibited good insecticidal activities against Mythimna separata Walker, Plutella xylostella (Linnaeus, 1758) and Laphygma exigua Hübner.
\end{abstract}

Keywords: amide; $N$-pyridylpyrazole; synthesis; crystal structure; insecticidal activity

\section{Introduction}

Development of crop-protection molecules with unique modes of action is necessary to combat widespread insecticide resistance. Calcium channels, in particular, the ryanodine receptor (RyR) represent an attractive biological target for insect control and thus offers excellent promise in integrated pest management strategies [1]. Anthranilic diamides, discovered by DuPont, are a promising novel class of insecticides which exhibit their action by binding to insect ryanodine receptors (RyR) and activating the uncontrolled release of calcium stores [2-4]. Anthranilic diamide 
insecticides are characterized by a three-part chemical structure as shown in Figure 1A, where $(\mathbf{X})$ is an anthraniloyl moiety, (Y) an aromatic acyl moiety and (Z) an aliphatic amide moiety. Notably, anthranilic diamides containing an $N$-pyridylpyrazole in the second section (Y) showed significantly better activity than other heterocyclic derivatives [5]. Work in this area has led to the discovery of Rynaxypyr $^{\mathrm{TM}}$ (Figure 1B), a highly potent and selective activator of insect ryanodine receptors with exceptional activity on a broad range of Lepidoptera, as the first new insecticide from this class [6].

Figure 1. Chemical structures of anthranilic diamide insecticides.

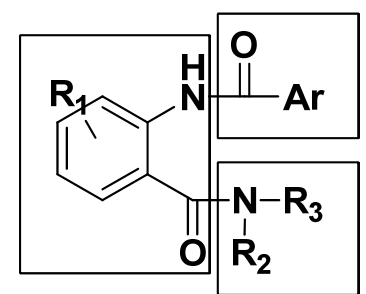

(X)
(Y)

(Z)

(A) General formula.<smiles>CNC(=O)c1cc(Cl)cc(C)c1NC(=O)c1cc(Br)nn1-c1ncccc1Cl</smiles>

(B) Rynaxypyr ${ }^{\mathrm{TM}}$.

In our previous work, when the $N$-pyridylpyrazole ring was replaced with 1,2,3-thiadiazole [7] or triazolopyrimidine [8] ones, the insecticidal activities were completely eliminated. In contrast, the modification of insecticidal anthranilic diamides with an ester group [9] or sulfonamide [10] substituting an amide group in the aliphatic amide moiety $(\mathbf{Z})$ showed similar insecticidal activity, though of a lesser degree. Thus, these results suggest that the $N$-pyridylpyrazole unit plays an important role in the insecticidal activities of anthranilic diamides, but the aliphatic amide moiety $(\mathbf{Z})$ may not be essential to insecticidal activities.

Encouraged by these reports, we developed an idea to examine whether the modification of the anthraniloyl skeleton by removing the aliphatic amide moiety $(\mathbf{Z})$ could have an effect on potential insecticidal activities. Enlightened by all of the descriptions above, to further explore the comprehensive structure-activity relationships of the insecticidal activity, a series of novel amides containing $\mathrm{N}$-pyridylpyrazoles were synthesized, and their insecticidal activities against Mythimna separata Walker, Culex pipiens pallens, Plutella xylostella (Linnaeus, 1758) and Laphygma exigua Hübner were tested and are discussed in this publication.

\section{Results and Discussion}

\subsection{Chemistry}

The synthetic route to the title compounds $\mathbf{7 a}-\mathbf{s}$ is shown in Scheme 1. The pyrazole carboxylic acid 6 is a key intermediate to the synthesis of target amides 7 containing $\mathrm{N}$-pyridylpyrazoles. Various synthetic routes have been reported for the synthesis of intermediate pyrazole-5-carboxylic acid 6 [11,12]. Considering the practical application of the synthetic method, an alternate route for the preparation of pyrazole carboxylic acid 6 was developed. Reaction of 2,3-dichloropyridine (1) with hydrazine hydrate at reflux using ethanol as solvent gave 3-chloro-2-hydrazinylpyridine (2). Condensation of diethyl maleate with hydrazine $\mathbf{2}$ in the presence of sodium ethoxide afforded the pyrazolidinone 3 . Subsequent treatment of $\mathbf{3}$ with phosphorus oxybromide in acetonitrile afforded the pyrazoline $\mathbf{4}$. 
A variety of reagents were explored for oxidation of $\mathbf{4}$ to the pyrazole $\mathbf{5}$. We first chose potassium permanganate as oxidant, but pyrazole 5 was obtained in only $32 \%$ yield. Subsequently potassium persulfate was used to give 5 in a good yield. The intermediate $\mathbf{6}$ could be prepared by hydrolysis of 5 with known methods. Finally, pyrazole carboxylic acid $\mathbf{6}$ was treated with oxalyl chloride at reflux to give the corresponding acid chloride, which was then reacted with commercially available substituted anilined to afford the title compounds $\mathbf{7 a}-\mathbf{s}$. The various amides $\mathbf{7 a}-\mathbf{s}$ containing $N$-pyridylpyrazoles prepared are listed in Table 1.

Scheme 1. The synthetic route to title compounds 7 .
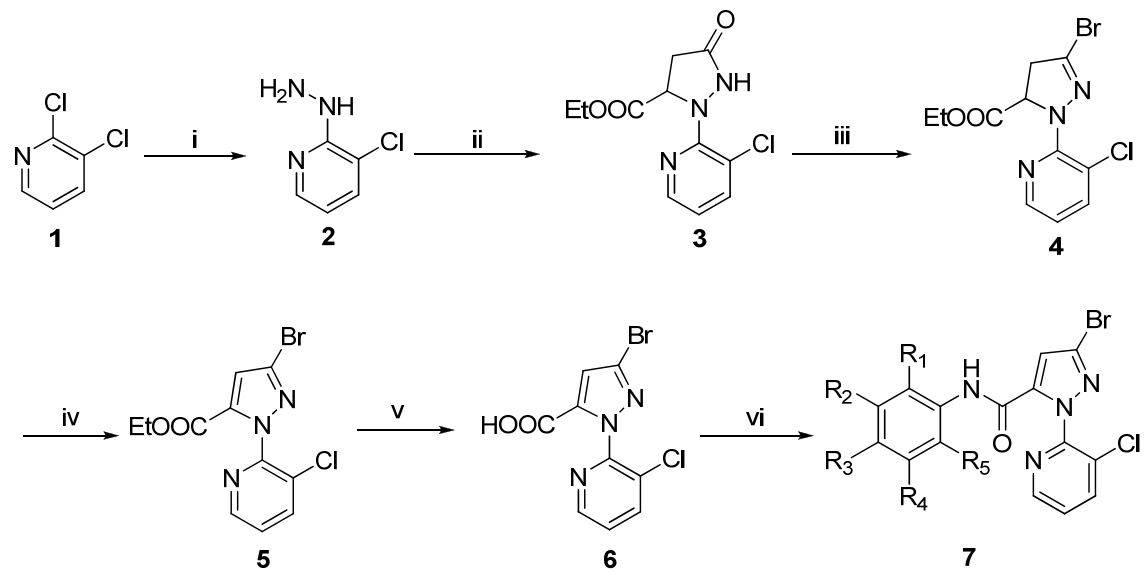

Reagents and conditions: (i) $\mathrm{N}_{2} \mathrm{H}_{4}, \mathrm{EtOH}$; (ii) Diethyl maleate, $\mathrm{NaOC}_{2} \mathrm{H}_{5} / \mathrm{C}_{2} \mathrm{H}_{5} \mathrm{OH}$; (iii) $\mathrm{POBr}_{3}$, $\mathrm{CH}_{3} \mathrm{CN}$; (iv) $\mathrm{K}_{2} \mathrm{~S}_{2} \mathrm{O}_{8}, \mathrm{H}_{2} \mathrm{SO}_{4}, \mathrm{CH}_{3} \mathrm{CN}$; (v) $\mathrm{NaOH}, \mathrm{MeOH}$; (vi) 1. $\mathrm{CH}_{2} \mathrm{Cl}_{2}$, oxalyl chloride, DMF 2. $\mathrm{ArNH}_{2}, \mathrm{CH}_{2} \mathrm{Cl}_{2},(i-P r)_{2} \mathrm{EtN}$.

Table 1. List of $N$-pyridylpyrazole-containing amides $\mathbf{7 a - s}$.<smiles>[R]c1c([R])c([R])c(NC(=O)c2cc(Br)nn2-c2ncccc2Cl)c([R3])c1[R]</smiles>

7a-s

\begin{tabular}{cccccccccccc}
\hline Compd. & $\mathbf{R}_{\mathbf{1}}$ & $\mathbf{R}_{\mathbf{2}}$ & $\mathbf{R}_{\mathbf{3}}$ & $\mathbf{R}_{\mathbf{4}}$ & $\mathbf{R}_{\mathbf{5}}$ & $\mathbf{C o m p d}$ & $\mathbf{R}_{\mathbf{1}}$ & $\mathbf{R}_{\mathbf{2}}$ & $\mathbf{R}_{\mathbf{3}}$ & $\mathbf{R}_{\mathbf{4}}$ & $\mathbf{R}_{\mathbf{5}}$ \\
\hline $\mathbf{7 a}$ & $\mathrm{H}$ & $\mathrm{Cl}$ & $\mathrm{H}$ & $\mathrm{H}$ & $\mathrm{H}$ & $\mathbf{7 k}$ & $\mathrm{CH}_{3}$ & $\mathrm{H}$ & $\mathrm{H}$ & $\mathrm{H}$ & $\mathrm{NO}_{2}$ \\
$\mathbf{7 b}$ & $\mathrm{H}$ & $\mathrm{H}$ & $\mathrm{F}$ & $\mathrm{H}$ & $\mathrm{H}$ & $\mathbf{7 1}$ & $\mathrm{Cl}$ & $\mathrm{H}$ & $\mathrm{NO}_{2}$ & $\mathrm{H}$ & $\mathrm{H}$ \\
$\mathbf{7 c}$ & $\mathrm{H}$ & $\mathrm{H}$ & $\mathrm{Cl}$ & $\mathrm{H}$ & $\mathrm{H}$ & $\mathbf{7 m}$ & $\mathrm{Br}$ & $\mathrm{H}$ & $\mathrm{NO}_{2}$ & $\mathrm{H}$ & $\mathrm{H}$ \\
$\mathbf{7 d}$ & $\mathrm{H}$ & $\mathrm{H}$ & $\mathrm{I}$ & $\mathrm{H}$ & $\mathrm{H}$ & $\mathbf{7 n}$ & $\mathrm{NO}_{2}$ & $\mathrm{H}$ & $\mathrm{Cl}$ & $\mathrm{H}$ & $\mathrm{H}$ \\
$\mathbf{7 e}$ & $\mathrm{H}$ & $\mathrm{H}$ & $\mathrm{NO}_{2}$ & $\mathrm{H}$ & $\mathrm{H}$ & $\mathbf{7 o}$ & $\mathrm{Cl}$ & $\mathrm{H}$ & $\mathrm{H}$ & $\mathrm{Cl}$ & $\mathrm{H}$ \\
$\mathbf{7 f}$ & $\mathrm{H}$ & $\mathrm{H}$ & $\mathrm{OC}_{2} \mathrm{H}_{5}$ & $\mathrm{H}$ & $\mathrm{H}$ & $\mathbf{7 p}$ & $\mathrm{CH}_{3}$ & $\mathrm{H}$ & $\mathrm{Cl}$ & $\mathrm{H}$ & $\mathrm{CH}_{3}$ \\
$\mathbf{7 g}$ & $\mathrm{H}$ & $\mathrm{Cl}$ & $\mathrm{F}$ & $\mathrm{H}$ & $\mathrm{H}$ & $\mathbf{7 q}$ & $\mathrm{CH}_{3}$ & $\mathrm{H}$ & $\mathrm{Br}$ & $\mathrm{H}$ & $\mathrm{CH}_{3}$ \\
$\mathbf{7 h}$ & $\mathrm{CH}_{3}$ & $\mathrm{H}$ & $\mathrm{CH}_{3}$ & $\mathrm{H}$ & $\mathrm{H}$ & $\mathbf{7 r}$ & $\mathrm{CH}_{3}$ & $\mathrm{H}$ & $\mathrm{NO}_{2}$ & $\mathrm{H}$ & $\mathrm{Cl}$ \\
$\mathbf{7 i}$ & $\mathrm{CH}_{3}$ & $\mathrm{H}$ & $\mathrm{NO}_{2}$ & $\mathrm{H}$ & $\mathrm{H}$ & $\mathbf{7 s}$ & $\mathrm{CH}_{3}$ & $\mathrm{H}$ & $\mathrm{Cl}$ & $\mathrm{H}$ & $\mathrm{NO}_{2}$ \\
$\mathbf{7 j}$ & $\mathrm{CH}_{3}$ & $\mathrm{H}$ & $\mathrm{H}$ & $\mathrm{H}$ & $\mathrm{CH}$ & & & & & & \\
\hline
\end{tabular}




\subsection{Crystal Structure}

The structure of compound $\mathbf{7 1}$ was further confirmed by single crystal X-ray diffraction analysis (Figures 2 and 3). In the molecular structure of title compound, the three ring (benzene ring, pyridine ring and pyrazole ring) are nearly vertically with $\theta$ angle of $80.6^{\circ}$ (benzene ring $v$ s. pyridine ring), $76.8^{\circ}$ (pyrazole ring vs. pyridine) respectively, but the pyrazole ring is planar with the benzene ring $\left(7.8^{\circ}\right)$.

Figure 2. The molecular structure of 71 .

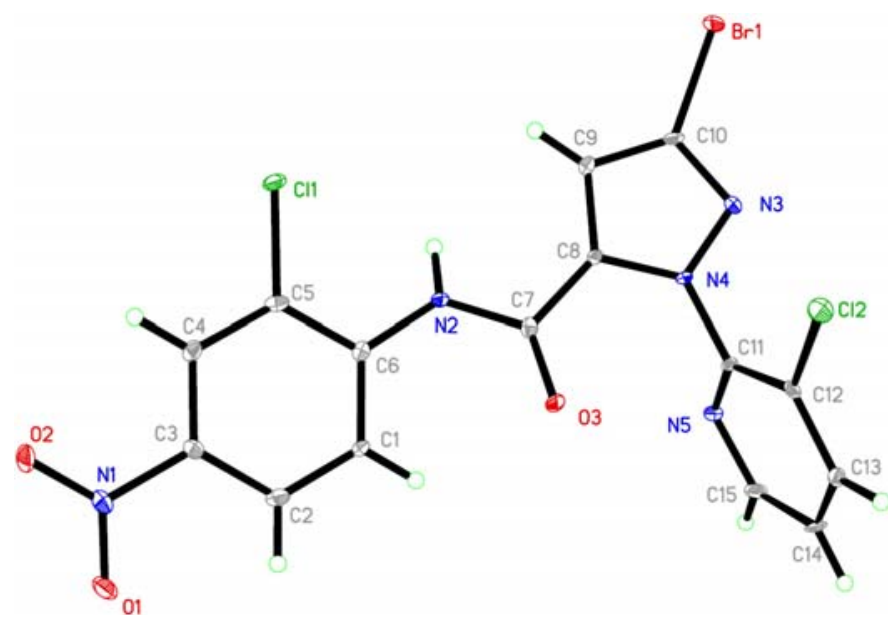

Figure 3. The packing of the molecules in the crystal lattice of 71.

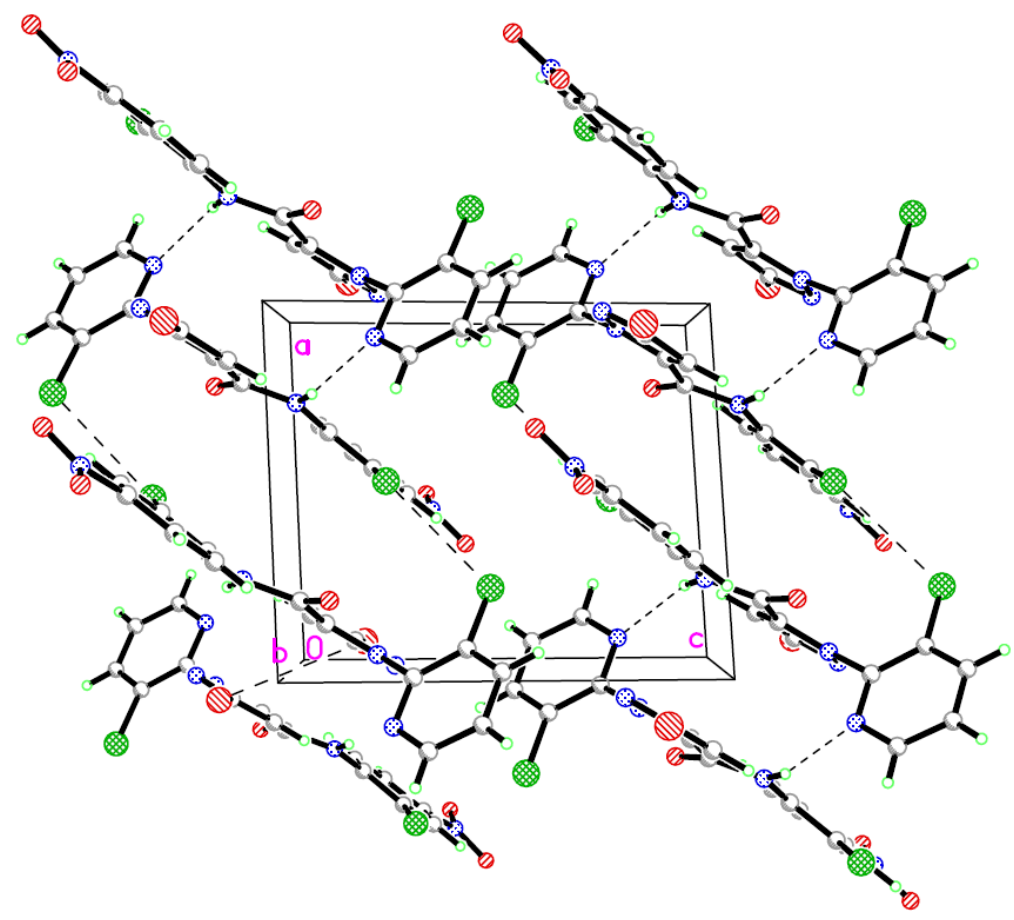

The average bond lengths and bond angles of the phenyl ring [13-17], the pyrazole ring [18], pyridine ring [19] and the amide bond [20-24] are normal. The intermolecular edge-to-face $\pi-\pi$ stacking appears between the pyridine ring and the phenyl ring in another adjacent molecule, in which the distance of $\mathrm{H} 13$ and the centroid of phenyl ring is $3.725 \AA$. These interactions can help to further stabilize the crystal structure. The title compound has an extensive network of hydrogen bonding 
involving the two acceptor $\mathrm{N}$ atoms. In the $b c$ plane, they are linked together by $\mathrm{N}-\mathrm{H} \cdots \mathrm{N}$ hydrogen bonds. This hydrogen-bonding sequence is repeated to form a ring.

\subsection{Insecticidal Activities and Structure-Activity Relationship (SAR)}

The insecticidal activities of all target compounds $7 \mathbf{a}-\mathbf{s}$ were determined in vivo. The results are summarized in Table 2. As shown, 71 showed the most potent insecticidal activity against oriental armyworm (M. separata) in all the tested compounds, the death rate is $80 \%$ at $10 \mu \mathrm{g} \cdot \mathrm{mL}^{-1}$. Compounds $\mathbf{7 c}, \mathbf{7 i}, \mathbf{7 p}, \mathbf{7 q}, \mathbf{7} \mathbf{i}$ and $\mathbf{7 r}$ also exhibited significant insecticidal activity against oriental armyworm, with death rates of more than $70 \%$ at $25 \mu \mathrm{g} \cdot \mathrm{mL}^{-1}$.

Table 2. Insecticidal activity against Mythimna separata Walker and Culex pipiens pallens of title compounds (mortality/\%).

\begin{tabular}{|c|c|c|c|c|c|c|c|}
\hline \multirow{3}{*}{ Compd. } & \multicolumn{6}{|c|}{ Mythimna separata Walker } & \multirow{3}{*}{$\begin{array}{c}\text { Culex pipiens pallens } \\
\mu \mathrm{g} \cdot \mathrm{mL}^{-1} / \text { death rate }(\%) \\
2\end{array}$} \\
\hline & \multicolumn{6}{|c|}{$\mu \mathrm{g} \cdot \mathrm{mL}^{-1} /$ death rate $(\%)$} & \\
\hline & 200 & 100 & $\mathbf{5 0}$ & 25 & 10 & 5 & \\
\hline $7 \mathbf{a}$ & 0 & & & & & & $-{ }^{a}$ \\
\hline $7 \mathrm{~b}$ & 100 & 100 & 100 & 50 & & & 0 \\
\hline $7 c$ & 100 & 100 & 100 & 70 & 0 & & 10 \\
\hline $7 d$ & 100 & 100 & 50 & & & & 100 \\
\hline $7 e$ & 100 & 90 & 20 & & & & 20 \\
\hline $7 f$ & 100 & 0 & & & & & 30 \\
\hline $7 \mathrm{~g}$ & 100 & 0 & & & & & - \\
\hline $7 \mathrm{~h}$ & 100 & 70 & 0 & & & & - \\
\hline $7 \mathbf{i}$ & 100 & 100 & 100 & 80 & 50 & & 40 \\
\hline $7 \mathbf{j}$ & 100 & 80 & 40 & & & & - \\
\hline $7 \mathbf{k}$ & 100 & 80 & 20 & & & & 50 \\
\hline 71 & 100 & 100 & 100 & 100 & 80 & 0 & 80 \\
\hline $7 \mathrm{~m}$ & 100 & 100 & 30 & & & & 40 \\
\hline $7 n$ & 10 & & & & & & 40 \\
\hline 70 & 0 & & & & & & 10 \\
\hline $7 p$ & 100 & 100 & 100 & 80 & 20 & & 30 \\
\hline $7 q$ & 100 & 100 & 100 & 100 & 30 & & 30 \\
\hline $7 r$ & 100 & 100 & 100 & 70 & 30 & & - \\
\hline $7 s$ & 100 & 100 & 100 & 60 & & & 20 \\
\hline Rynaxypyr $^{\mathrm{TM}}$ & & & & & & 100 & 100 \\
\hline
\end{tabular}

Further, the Structure-Activity Relationships (SAR) for different substitutions on the phenyl ring can be inferred from the results. Compound $7 \mathbf{i}$ with an electron-donating methyl group at $\mathrm{R}^{1}$ exhibited similar insecticidal activity to that of compound $\mathbf{7 l}$ with an electron-withdrawing chlorine group at $\mathrm{R}^{1}$, but the insecticidal activity decreased significantly when the chlorine atom was replaced with a bromine atom at $\mathrm{R}^{1}$ (compound $\mathbf{7 m}$ ). From these results, it appears that steric effects rather than electrostatic effects have substantial effects on the insecticidal activity at the $\mathrm{R}^{1}$. This observation appears consistent with the structure-activity of anthranilic diamides reported in the literature [25]. Among 
compounds $\mathbf{7 b}-\mathbf{e}$, the electron-withdrawing group substituted analogues at $\mathrm{R}^{3}$ (compounds $\mathbf{7 b}-\mathbf{e}$ ) were more active than the electron-donating group substituted analogue (compound 7f). Besides, the electron-withdrawing halide substituted analogues at $\mathrm{R}^{3}$ (compounds $\mathbf{7 p}, \mathbf{7 q}, \mathbf{7 s}$ ) exhibited more insecticidal potency than unsubstituted analogs (compounds $\mathbf{7 j}, \mathbf{7 k}$ ). The electron-donating group substitution (compound $\mathbf{7 j}$ ) and electron-withdrawing group substitution (compound $\mathbf{7 k}$ ) showed similar levels of activity, and the presence of a chloro substituent at $\mathrm{R}^{5}$ (compound $\mathbf{7 r}$ ) and unsubstituted analog (compound 7i) exhibited similar insecticidal activity. This result indicates that different substitutions at $\mathrm{R}^{5}$ did not exhibit significant influence on insecticidal activities. In addition, the insecticidal activity decreased significantly when electron-withdrawing chloro substituent was present at $\mathrm{R}^{2}$ and $\mathrm{R}^{4}$, such as 7a, 7g, 7o. As shown in Table 2, the title compounds displayed good larvicidal activities against $C$. p. pallens, for example, the larvicidal activity of $\mathbf{7 d}$ was $100 \%$ at $2 \mu \mathrm{g} \cdot \mathrm{mL}^{-1}$, as compared with $100 \%$ mortality of Rynaxypyr ${ }^{\mathrm{TM}}$ at the same concentration, but showed no clear SAR trends.

Figure 4 shows the symptoms of larvae affected by the title compounds and commercial Rynaxypyr $^{\mathrm{TM}}$. Insects treated with the title compound 71 showed abnormal symptoms such as body contraction, vomiting, feeding cessation, body thickening and shortening, which are similar to those observed for larvae treated with commercial Rynaxypyr ${ }^{\mathrm{TM}}$. These results suggest that the title compounds exhibit their activity by activating insect RyR.

Figure 4. Symptoms of forth-instar larvae of Mythimna separata Walker treated by leaf dipping.

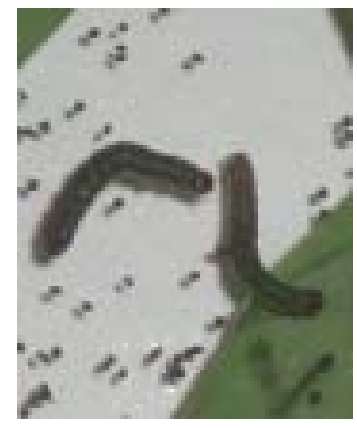

(a)

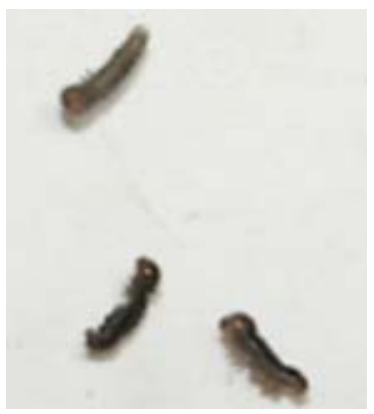

(b)

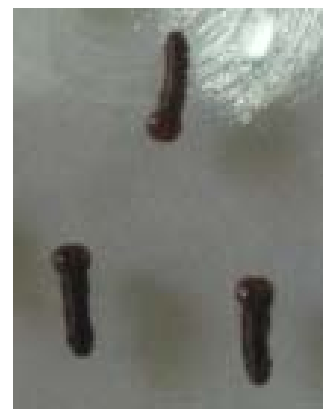

(c)

(a) Untreated; (b) Rynaxypyr ${ }^{\mathrm{TM}}$ at $10 \mu \mathrm{g} \cdot \mathrm{mL}^{-1}, 36 \mathrm{~h}$ after application; (c) 71 at $10 \mu \mathrm{g} \cdot \mathrm{mL}^{-1}, 36 \mathrm{~h}$ after application.

For the compounds $\mathbf{7 b}, \mathbf{7 c}, \mathbf{7 p}, \mathbf{7 q}, \mathbf{7 s}, \mathbf{7}, \mathbf{7 m}$, further bioassay was conducted against $P$. xylostella and L. exigua. The results are summarized in Table 3. At a dose of $25 \mu \mathrm{g} \cdot \mathrm{mL}^{-1}$, most of compounds have good insecticidal activity against $P$. xylostella and L. exigua, which can be compared with that of the control Rynaxypyr ${ }^{\mathrm{TM}}$. This result showed that the title compounds have insecticidal activity on a broad spectrum of Lepidoptera. 
Table 3. Insecticidal activity against Plutella xylostella (Linnaeus, 1758) and Laphygma exigua Hübner of title compounds (mortality/\%).

\begin{tabular}{|c|c|c|c|c|c|c|c|c|}
\hline \multirow{3}{*}{ Compd. } & \multirow{2}{*}{\multicolumn{4}{|c|}{$\begin{array}{c}\text { Plutella xylostella }(\text { Linnaeus, 1758) } \\
\mu \mathrm{g} \cdot \mathrm{mL}^{-1} / \text { death rate }(\%)\end{array}$}} & \multirow{2}{*}{\multicolumn{4}{|c|}{$\begin{array}{c}\text { Laphygma exigua Hübner } \\
\mu \mathrm{g} \cdot \mathrm{mL}^{-1} / \text { death rate }(\%)\end{array}$}} \\
\hline & & & & & & & & \\
\hline & 200 & 100 & 50 & 25 & 200 & 100 & 50 & 25 \\
\hline $7 b$ & 100 & 98 & 99 & 88 & 95 & 100 & 92 & 96 \\
\hline $7 \mathrm{c}$ & 100 & 98 & 100 & 97 & 100 & 100 & 100 & 100 \\
\hline $7 \mathrm{~m}$ & 100 & 98 & 96 & 90 & 100 & 100 & 100 & 100 \\
\hline 71 & 78 & 72 & 0 & & 91 & 86 & 78 & 65 \\
\hline $7 p$ & 100 & 100 & 97 & 94 & 100 & 100 & 98 & 94 \\
\hline $7 q$ & 89 & 72 & 0 & $-^{a}$ & 100 & 100 & 89 & 93 \\
\hline $7 s$ & 95 & 89 & 84 & 76 & 98 & 100 & 95 & 92 \\
\hline Rynaxypyr $^{\mathrm{TM}}$ & - & - & 100 & 94 & - & - & 100 & 100 \\
\hline $\mathbf{C k}$ & 0 & 0 & 0 & 0 & 0 & 0 & 0 & 0 \\
\hline
\end{tabular}

${ }^{a}$ Not tested.

\section{Experimental}

\subsection{General}

Melting points were determined on an X-4 binocular microscope melting point apparatus and were uncorrected. ${ }^{1} \mathrm{H}-\mathrm{NMR}$ spectra were obtained on a Bruker AC-P500 spectrometer or Bruker Avance 400 spectrometer using tetramethylsilane (TMS) as an internal standard and $\mathrm{CDCl}_{3}$ or DMSO- $d_{6}$ as solvents. Elemental analyses were performed on a Vario EL elemental analyzer. High-resolution mass (HRMS) were recorded on a 7.0-T (Ionspec, Irvine, CA, USA) Fourier transform ion cyclotron resonance mass spectrometer. Crystallographic data of the compound $\mathbf{7 1}$ were collected on a Rigaku Saturn diffractometer. All chemicals or reagents were purchased from standard commercial suppliers. Petroleum ether refers to the fraction of bp $60-90{ }^{\circ} \mathrm{C}$.

\subsection{Chemical Synthesis}

\subsubsection{3-Chloro-2-hydrazinylpyridine (2)}

$50 \%$ Hydrazine hydrate $(200 \mathrm{~mL})$.was added to a suspension of 2,3-dichloropyridine $(1,73.5 \mathrm{~g}$, $0.5 \mathrm{~mol})$ in anhydrous ethanol $(300 \mathrm{~mL})$. The resulting mixture was refluxed for $36 \mathrm{~h}$, and then cooled to room temperature. A white crystalline product precipitated out of solution, which was collected by filtration, washed thoroughly with cold ethanol and dried to give hydrazine 2 (49.6 g, 69.0\%), m.p. 163-164 ${ }^{\circ} \mathrm{C} ;{ }^{1} \mathrm{H}-\mathrm{NMR}\left(\mathrm{CDCl}_{3}, 400 \mathrm{MHz}\right) \delta$ : 3.97 (br s, 2H, NH ), 6.21 (br s, $\left.1 \mathrm{H}, \mathrm{NH}\right), 6.64$ (m, 1H, pyridyl-H), 7.47 (d, $J=7.6 \mathrm{~Hz}, 1 \mathrm{H}$, pyridyl-H), 8.09 (d, $J=4.9 \mathrm{~Hz}, 1 \mathrm{H}$, pyridyl-H).

\subsubsection{Ethyl 2-(3-Chloro-2-pyridinyl)-5-oxopyrazolidine-3-carboxylate (3)}

To absolute ethanol $(200 \mathrm{~mL})$ in a $500 \mathrm{~mL}$ three-necked round-bottomed flask was added sodium $(6.9 \mathrm{~g}, 0.3 \mathrm{~mol})$ cut into pieces of suitable size. After all the sodium had reacted, the mixture was heated to reflux and $2(39.82 \mathrm{~g}, 0.277 \mathrm{~mol})$ was added. The mixture was refluxed for $10 \mathrm{~min}$, then 
diethyl maleate $(51.65 \mathrm{~g}, 0.3 \mathrm{~mol})$ was added dropwise. The resulting orange-red solution was held at reflux for $30 \mathrm{~min}$. After being cooled to $65^{\circ} \mathrm{C}$, the reaction mixture was treated with glacial acetic acid $(30 \mathrm{~g}, 0.51 \mathrm{~mol})$. The mixture was diluted with water $(30 \mathrm{~mL})$. After removal of most of the solvent, the residue was treated with water $(300 \mathrm{~mL})$. The slurry formed was dissolved in aqueous ethanol $(70 \%, 200 \mathrm{~mL})$ and stirred thoroughly. The solid was collected by filtration, washed with aqueous ethanol $(50 \%, 50 \mathrm{~mL} \times 3)$ to give pyrazolidinone $3(36.6 \mathrm{~g}, 49.0 \%)$, m.p. $132-134{ }^{\circ} \mathrm{C}$; ${ }^{1} \mathrm{H}-\mathrm{NMR}$ (DMSO- $\left.d_{6}, 300 \mathrm{MHz}\right) \delta: 1.20\left(\mathrm{t}, J=6.8 \mathrm{~Hz}, 3 \mathrm{H}, \mathrm{CH}_{2} \mathrm{CH}_{3}\right), 4.18\left(\mathrm{q}, J=7.2 \mathrm{~Hz}, 2 \mathrm{H}, \mathrm{CH}_{2} \mathrm{CH}_{3}\right), 2.34$ (d, $\left.J=16.8 \mathrm{~Hz}, 1 \mathrm{H}, \mathrm{CH}_{2}\right), 2.90(\mathrm{q}, J=10.0 \mathrm{~Hz}, 1 \mathrm{H}, \mathrm{CH}), 4.81(\mathrm{~d}, J=9.2 \mathrm{~Hz}, 1 \mathrm{H}, \mathrm{CH} 2), 7.18(\mathrm{dd}, J=4.8$, $7.6 \mathrm{~Hz}, 1 \mathrm{H}$, pyridyl-H), 7.92 (d, $J=7.6 \mathrm{~Hz}, 1 \mathrm{H}$, pyridyl-H), 8.25 (d, $J=4.0 \mathrm{~Hz}, 1 \mathrm{H}$, pyridyl-H), 10.18 (br s, 1H, NH).

\subsubsection{Ethyl 3-Bromo-1-(3-chloro-2-pyridinyl)-4,5-dihydro-1H-pyrazole-5-carboxylate (4)}

To a solution of $3(0.1 \mathrm{~mol})$ in acetonitrile $(300 \mathrm{~mL})$ was added phosphorus oxybromide $(0.12 \mathrm{mmol})$. The reaction mixture was refluxed for $5 \mathrm{~h}$, then most of the solvent (ca. $250 \mathrm{~mL}$ ) was removed by distillation. The concentrated reaction mixture was slowly poured into saturated aq. $\mathrm{Na}_{2} \mathrm{CO}_{3}(250 \mathrm{~mL})$ and stirred vigorously for $30 \mathrm{~min}$. The resulting mixture was extracted with $\mathrm{CH}_{2} \mathrm{Cl}_{2}(250 \mathrm{~mL} \times 2)$, the organic extract was separated, dried, filtered, concentrated and purified by silica gel chromatography to afford intermediates 4. Yield 93.0\%, m.p. 59-60 ${ }^{\circ} \mathrm{C}$; ${ }^{1} \mathrm{H}-\mathrm{NMR}\left(\mathrm{DMSO}-d_{6}, 400 \mathrm{MHz}\right) \delta$ : $1.12(\mathrm{t}$, $\left.J=7.0 \mathrm{~Hz}, 3 \mathrm{H}, \mathrm{CH}_{2} \mathrm{CH}_{3}\right), 3.24-3.31\left(\mathrm{~m}, 1 \mathrm{H}, \mathrm{CH}_{2}\right), 3.54-3.61\left(\mathrm{~m}, 1 \mathrm{H}, \mathrm{CH}_{2}\right), 4.08(\mathrm{q}, J=7.0 \mathrm{~Hz}, 2 \mathrm{H}$, $\left.\mathrm{CH}_{2} \mathrm{CH}_{3}\right), 5.14-5.19(\mathrm{~m}, 1 \mathrm{H}, \mathrm{CH}), 6.98$ (dd, $J=4.8,7.6 \mathrm{~Hz}, 1 \mathrm{H}$, pyridyl-H), 7.83 (d, $J=7.7 \mathrm{~Hz}, 1 \mathrm{H}$, pyridyl-H), 8.10 (d, $J=4.4 \mathrm{~Hz}, 1 \mathrm{H}$, pyridyl-H).

\subsubsection{Ethyl 3-Bromo-1-(3-chloro-2-pyridinyl)-1H-pyrazole-5-carboxylate (5)}

To a solution of $4(51 \mathrm{mmol})$ in acetonitrile $(250 \mathrm{~mL})$ was added sulfuric acid $(98 \%, 10 \mathrm{~g}, 102 \mathrm{mmol})$. After being stirred for several minutes, the reaction mixture was treated with $\mathrm{K}_{2} \mathrm{~S}_{2} \mathrm{O}_{8}(21 \mathrm{~g}, 76.5 \mathrm{mmol})$ and refluxed for $4.5 \mathrm{~h}$. After being cooled to $60{ }^{\circ} \mathrm{C}$, the mixture was filtered, the filter cake was washed with acetonitrile $(30 \mathrm{~mL})$. The filtrate was concentrated to $100 \mathrm{~mL}$, then added slowly to water $(250 \mathrm{~mL})$ under stirring. The solid was collected by filtration, washed with acetonitrile $(25 \%, 30 \mathrm{~mL} \times 3)$, water $(30 \mathrm{~mL})$, and then dried to give intermediates 5. Yield $92.7 \%$, m.p. $117-118{ }^{\circ} \mathrm{C}$; ${ }^{1} \mathrm{H}-\mathrm{NMR}$ $\left(\mathrm{CDCl}_{3}, 400 \mathrm{MHz}\right) \delta: 1.21\left(\mathrm{t}, J=6.8 \mathrm{~Hz}, 3 \mathrm{H}, \mathrm{CH}_{2} \mathrm{CH}_{3}\right), 4.22\left(\mathrm{q}, J=7.2 \mathrm{~Hz}, 2 \mathrm{H}, \mathrm{CH}_{2} \mathrm{CH}_{3}\right), 7.03(\mathrm{~s}, 1 \mathrm{H}$, pyrazolyl-H), 7.44 (dd, $J=4.8,8.4 \mathrm{~Hz}, 1 \mathrm{H}$, pyridyl-H), 7.91 (dd, $J=1.4,8.0 \mathrm{~Hz}, 1 \mathrm{H}$, pyridyl-H), 8.51 (dd, $J=1.4,4.7 \mathrm{~Hz}, 1 \mathrm{H}$, pyridyl-H).

\subsubsection{3-Bromo-1-(3-chloro-2-pyridinyl)-1H-pyrazole-5-carboxylic acid (6)}

A mixture of $5(47.2 \mathrm{mmol})$, methanol $(120 \mathrm{~mL}), \mathrm{H}_{2} \mathrm{O}(60 \mathrm{~mL})$ and $\mathrm{NaOH}(2.3 \mathrm{~g}, 56.6 \mathrm{mmol})$ was stirred at room temperature for $6 \mathrm{~h}$, then concentrated in vacuo to about $80 \mathrm{~mL}$. The concentrated mixture was diluted with $\mathrm{H}_{2} \mathrm{O}(150 \mathrm{~mL})$, and washed with ethyl acetate $(150 \mathrm{~mL})$. The aqueous solution was acidified using concentrated hydrochloric acid to $\mathrm{pH} 1.5$. The solid was collected by filtration, washed with water $(30 \mathrm{~mL})$, and then dried to give pyrazolecarboxylic acid 6 . Yield $89.3 \%$, 
m.p. $197-200{ }^{\circ} \mathrm{C} ;{ }^{1} \mathrm{H}-\mathrm{NMR}\left(\mathrm{CDCl}_{3}, 300 \mathrm{MHz}\right) \delta$ : 7.10 (s, 1H, pyrazolyl-H), $7.48(\mathrm{dd}, J=4.8,8.1 \mathrm{~Hz}$, $1 \mathrm{H}$, pyridyl-H), 7.94 (dd, $J=1.4,8.0 \mathrm{~Hz}, 1 \mathrm{H}$, pyridyl-H), 8.52 (dd, $J=1.4,4.7 \mathrm{~Hz}, 1 \mathrm{H}$, pyridyl-H).

\subsubsection{General Procedure for the Synthesis of Compounds $\mathbf{7 a - s}$}

To a suspension of $N$-pyridylpyrazole acid $6(1 \mathrm{mmol})$ in dichloromethane $(20 \mathrm{~mL})$ was added oxalyl chloride ( $3 \mathrm{mmol}$ ), followed by dimethylformamide ( 2 drops). The solution was stirred at room temperature. After $3 \mathrm{~h}$ the mixture was concentrated in vacuo to obtain the crude acid chloride. The crude acid chloride in dichloromethane $(10 \mathrm{~mL})$ was added slowly to a stirred solution of substituted aniline $1(1.2 \mathrm{mmol})$ in dichloromethane $(20 \mathrm{~mL})$ in an ice bath. After $20 \mathrm{~min}$, diisopropylethylamine $(1 \mathrm{mmol})$ was added dropwise. The solution was warmed to room temperature and stirred for $12 \mathrm{~h}$, then diluted with $\mathrm{CH}_{2} \mathrm{Cl}_{2}(20 \mathrm{~mL})$, and washed with $1 \mathrm{~mol} \cdot \mathrm{L}^{-1}$ aq. $\mathrm{HCl}$ solution $(20 \mathrm{~mL})$, saturated aq. $\mathrm{NaHCO}_{3}(20 \mathrm{~mL})$, and brine $(20 \mathrm{~mL})$. The organic extract was separated, dried, filtered, concentrated and purified by silica gel chromatography to afford the desired $N$-pyridylpyrazole-containing amides 7 .

3-Bromo-N-(3-chlorophenyl)-1-(3-chloropyridin-2-yl)-1H-pyrazole-5-carboxamide (7a). Yield: 70.7\%. White solid, m.p. $154-156{ }^{\circ} \mathrm{C} ;{ }^{1} \mathrm{H}-\mathrm{NMR}\left(\mathrm{CDCl}_{3}, 400 \mathrm{MHz}\right) \delta: 6.87$ (s, 1H, pyrazolyl-H), 7.10-7.14 (m, $3 \mathrm{H}, \mathrm{Ar}-\mathrm{H}), 7.44$ (dd, $J=4.8,8.0 \mathrm{~Hz}, 1 \mathrm{H}$, pyridyl-H), 7.58-7.59 (m, 1H, Ar-H), 7.93 (dd, $J=1.6,8.0 \mathrm{~Hz}$, $1 \mathrm{H}$, pyridyl-H), 8.26 (br. s, NH), 8.48 (dd, $J=1.6,4.8 \mathrm{~Hz}, 1 \mathrm{H}$, pyridyl-H); Elemental anal. (\%), calcd. for $\mathrm{C}_{15} \mathrm{H}_{9} \mathrm{BrCl}_{2} \mathrm{~N}_{4} \mathrm{O}$ : C, 43.72; H, 2.20; N, 13.60; found: C, 43.56; H, 2.55; N, 13.30 .

3-Bromo-1-(3-chloropyridin-2-yl)-N-(4-fluorophenyl)-1H-pyrazole-5-carboxamide (7b). Yield: 86.4\%. White solid, m.p. $197-198{ }^{\circ} \mathrm{C} ;{ }^{1} \mathrm{H}-\mathrm{NMR}\left(\mathrm{CDCl}_{3}, 400 \mathrm{MHz}\right) \delta: 6.84$ (s, 1H, pyrazolyl-H), 6.93-6.97 (m, 2H, Ar-H), 7.34-7.37 (m, 2H, Ar-H), 7.41 (dd, $J=4.8,8.0 \mathrm{~Hz}, 1 \mathrm{H}$, pyridyl-H), 7.91 (dd, $J=1.6,8.0 \mathrm{~Hz}$, $1 \mathrm{H}$, pyridyl-H), 8.43-8.45 (m, 2H, pyridyl-H, NH); Elemental anal. (\%), calcd. for $\mathrm{C}_{15} \mathrm{H}_{9} \mathrm{BrClFN}_{4} \mathrm{O}: \mathrm{C}$, 45.54; H, 2.29; N, 14.16; found: C, 45.65; H, 2.58; N, 13.90 .

3-Bromo-N-(4-chlorophenyl)-1-(3-chloropyridin-2-yl)-1H-pyrazole-5-carboxamide (7c). Yield: 79.9\%. White solid, m.p. ${ }^{179-180}{ }^{\circ} \mathrm{C} ;{ }^{1} \mathrm{H}-\mathrm{NMR}\left(\mathrm{CDCl}_{3}, 400 \mathrm{MHz}\right) \delta: 6.87$ (s, 1H, pyrazolyl-H), 6.23-6.26 (m, 2H, Ar-H), 7.37-7.40 (m, 2H, Ar-H), 7.43 (dd, $J=4.8,8.0 \mathrm{~Hz}, 1 \mathrm{H}$, pyridyl-H), 7.93 (dd, $J=1.6,8.0 \mathrm{~Hz}$, $1 \mathrm{H}$, pyridyl-H), 8.27 (br. s, NH), 8.47 (dd, 1H, $J=1.6,4.8 \mathrm{~Hz}$, pyridyl-H); Elemental anal. (\%), calcd. for $\mathrm{C}_{15} \mathrm{H}_{9} \mathrm{BrCl}_{2} \mathrm{~N}_{4} \mathrm{O}$ : C, 43.72; H, 2.20; N, 13.60; found: C, 43.82; H, 2.29; N, 13.53.

3-Bromo-1-(3-chloropyridin-2-yl)-N-(4-iodophenyl)-1H-pyrazole-5-carboxamide (7d). Yield: 62.3\%.

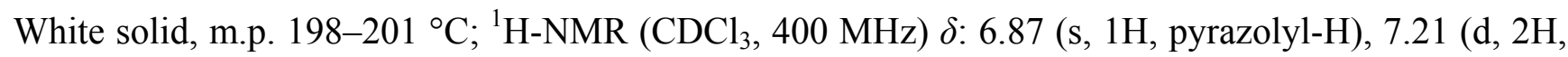
$J=8.8 \mathrm{~Hz}$, Ar-H), 7.45 (dd, $J=4.8,8.0 \mathrm{~Hz}, 1 \mathrm{H}$, pyridyl-H), 7.58 (d, 2H, $J=8.4 \mathrm{~Hz}, \mathrm{Ar}-\mathrm{H}), 7.94$ (dd, $1 \mathrm{H}, J=1.6,8.0 \mathrm{~Hz}$, pyridyl-H), 8.275 (br. s, $\mathrm{NH}$ ), 8.48 (dd, $1 \mathrm{H}, J=1.6,4.8 \mathrm{~Hz}$, pyridyl-H); Elemental anal. (\%), calcd. for $\mathrm{C}_{15} \mathrm{H}_{9} \mathrm{BrClIN}_{4} \mathrm{O}: \mathrm{C}, 35.78 ; \mathrm{H}, 1.80 ; \mathrm{N}, 11.13$; found: $\mathrm{C}, 36.14 ; \mathrm{H}, 2.21 ; \mathrm{N}, 10.82$.

3-Bromo-1-(3-chloropyridin-2-yl)-N-(4-nitrophenyl)-1H-pyrazole-5-carboxamide (7e). Yield: 64.5\%. White solid, m.p. 240-243 ${ }^{\circ} \mathrm{C}$; 1H-NMR (CDCl3, $\left.400 \mathrm{MHz}\right) \delta: 7.00$ (s, 1H, pyrazolyl-H), 7.48 (dd, $J=4.8,8.0 \mathrm{~Hz}, 1 \mathrm{H}$, pyridyl-H), 7.69-7.71 (m, 2H, Ar-H), 7.96 (d, 1H, $J=8.0 \mathrm{~Hz}$, pyridyl-H), 8.19-8.21 (m, 2H, Ar-H), 8.51 (d, 1H, $J=4.8 \mathrm{~Hz}$, pyridyl-H), 8.70 (br. s, NH); Elemental anal. (\%), calcd. for $\mathrm{C}_{15} \mathrm{H}_{9} \mathrm{BrClN}_{5} \mathrm{O}_{3}: \mathrm{C}, 42.63 ; \mathrm{H}, 2.15 ; \mathrm{N}, 16.57$; found: $\mathrm{C}, 42.91 ; \mathrm{H}, 2.48 ; \mathrm{N}, 16.51$. 
3-Bromo-1-(3-chloropyridin-2-yl)-N-(4-ethoxyphenyl)-1H-pyrazole-5-carboxamide (7f). Yield: 90.9\%. White solid, m.p. ${ }^{191-193}{ }^{\circ} \mathrm{C} ;{ }^{1} \mathrm{H}-\mathrm{NMR}\left(\mathrm{CDCl}_{3}, 400 \mathrm{MHz}\right) \delta: 1.39$ (t, 3H, J=6.8 Hz, CH ), 3.98 (q, 2H, $\left.J=6.8 \mathrm{~Hz}, \mathrm{CH}_{2}\right), 6.79-6.81$ (m, 2H, Ar-H), 6.84 (s, 1H, pyrazolyl-H), 7.31-7.33 (m, 2H, Ar-H), 7.41 (dd, $J=4.4,8.0 \mathrm{~Hz}, 1 \mathrm{H}$, pyridyl-H), 7.91 (dd, $J=1.6,8.0 \mathrm{~Hz}, 1 \mathrm{H}$, pyridyl-H), 8.15 (br. s, NH), 8.46 (dd, $1 \mathrm{H}, J=1.6,4.8 \mathrm{~Hz}$, pyridyl-H); Elemental anal. (\%), calcd. for $\mathrm{C}_{17} \mathrm{H}_{14} \mathrm{BrClN}_{4} \mathrm{O}_{2}: \mathrm{C}, 48.42 ; \mathrm{H}, 3.35 ; \mathrm{N}$, 13.29; found: C, 48.66; H, 3.20; N, 12.91 .

3-Bromo-N-(3-chloro-4-fluorophenyl)-1-(3-chloropyridin-2-yl)-1H-pyrazole-5-carboxamide (7g). Yield: 95.3\%. White solid, m.p. 167-169 ${ }^{\circ} \mathrm{C}$; ${ }^{1} \mathrm{H}-\mathrm{NMR}\left(\mathrm{CDCl}_{3}, 400 \mathrm{MHz}\right) \delta$ : 6.85 (s, $1 \mathrm{H}$, pyrazolyl-H), 7.02-7.07 (m, 2H, Ar-H), 7.20-7.23 (m, 2H, Ar-H), 7.45 (dd, J=4.8, 7.6 Hz, 1H, pyridyl-H), 7.63 (dd, $1 \mathrm{H}, J=1.6,5.6 \mathrm{~Hz}$, pyridyl-H), 7.95 (d, $1 \mathrm{H}, J=8.0 \mathrm{~Hz}$, pyridyl-H), 8.35 (br. s, NH); Elemental anal. (\%), calcd. for $\mathrm{C}_{15} \mathrm{H}_{8} \mathrm{BrCl}_{2} \mathrm{FN}_{4} \mathrm{O}$ : C, 41.89; H, 1.87; N, 13.03; found: C, 41.63; H, 2.17; N, 12.74 .

3-Bromo-1-(3-chloropyridin-2-yl)-N-(2,4-dimethylphenyl)-1H-pyrazole-5-carboxamide (7h). Yield: 69.1\%. White solid, m.p. $168-170{ }^{\circ} \mathrm{C} ;{ }^{1} \mathrm{H}-\mathrm{NMR}\left(\mathrm{CDCl}_{3}, 400 \mathrm{MHz}\right) \delta: 2.20\left(\mathrm{~s}, 3 \mathrm{H}, \mathrm{CH}_{3}\right), 2.27$ (s, 3H, $\left.\mathrm{CH}_{3}\right), 6.85$ (s, 1H, pyrazolyl-H), 6.96-7.00 (m, 2H, Ar-H), 7.39 (dd, 1H, J=4.8, 8.0 Hz, pyridyl-H), 7.46 (d, $1 \mathrm{H}, J=8.0 \mathrm{~Hz}, \mathrm{Ar}-\mathrm{H}), 7.66$ (br. s, $1 \mathrm{H}, \mathrm{NH}$ ), 7.87 (dd, 1H, $J=1.2,8.0 \mathrm{~Hz}$, pyridyl-H), 8.46 (dd, $1 \mathrm{H}, J=1.6,4.8 \mathrm{~Hz}$, pyridyl-H; Elemental anal. (\%), calcd. for $\mathrm{C}_{17} \mathrm{H}_{14} \mathrm{BrClN} \mathrm{O}_{4} \mathrm{O}$ C, 50.33; H, 3.48; N, 13.81; found: C, 50.63; H, 3.50; N, 13.75 .

3-Bromo-1-(3-chloropyridin-2-yl)-N-(2-methyl-4-nitrophenyl)-1H-pyrazole-5-carboxamide (7i). Yield: 62.3\%. Yellow solid, m.p. 185-187 ${ }^{\circ} \mathrm{C}$; ${ }^{1} \mathrm{H}-\mathrm{NMR}$ (DMSO- $\left.d_{6}, 400 \mathrm{MHz}\right) \delta: 2.35$ (s, 3H, $\mathrm{CH}_{3}$ ), 7.49 (s, 1H, Het-H), 7.61-7.67 (m, 2H, Ar-H), 8.06 (dd, $J=4.2 \mathrm{~Hz}, J=4.4 \mathrm{~Hz}, 1 \mathrm{H}, \mathrm{Ar}-\mathrm{H}), 8.17$ (d, $J=3.8 \mathrm{~Hz}$, $1 \mathrm{H}, \mathrm{Py}-\mathrm{H}), 8.22$ (d, $J=4.8 \mathrm{~Hz}, 1 \mathrm{H}, \mathrm{Py}-\mathrm{H}), 8.53$ (dd, $J=4.5 \mathrm{~Hz}, J=1.5 \mathrm{~Hz}, 1 \mathrm{H}, \mathrm{Py}-\mathrm{H}), 10.57(\mathrm{~s}, 1 \mathrm{H}$, $\mathrm{NH}$ ); Elemental anal. (\%), calcd. for $\mathrm{C}_{16} \mathrm{H}_{11} \mathrm{BrClN}_{5} \mathrm{O}_{3}: \mathrm{C}, 44.01 ; \mathrm{H}, 2.54 ; \mathrm{N}, 16.04$; found: $\mathrm{C}, 44.23$; $\mathrm{H}$, $2.38 ; \mathrm{N}, 15.89$.

3-Bromo-1-(3-chloropyridin-2-yl)-N-(2,6-dimethylphenyl)-1H-pyrazole-5-carboxamide (7j). Yield: 49.5\%. White solid, m.p. $216-219{ }^{\circ} \mathrm{C} ;{ }^{1} \mathrm{H}-\mathrm{NMR}\left(\mathrm{CDCl}_{3}, 400 \mathrm{MHz}\right) \delta: 2.21\left(\mathrm{~s}, 6 \mathrm{H}, \mathrm{CH}_{3}\right), 6.93(\mathrm{~s}, 1 \mathrm{H}$, pyrazolyl-H), 7.05-7.13 (m, 3H, Ar-H), 7.36-7.39 (m, 1H, pyridyl-H, 1H, NH), 7.87 (d, 1H, $J=6.8 \mathrm{~Hz}$, pyridyl-H), 8.46 (d, $1 \mathrm{H}, J=1.6,4.4 \mathrm{~Hz}$, pyridyl-H); Elemental anal. (\%), calcd. for $\mathrm{C}_{17} \mathrm{H}_{14} \mathrm{BrClN}_{4} \mathrm{O}$ : C, 50.33; H, 3.48; N, 13.81; found: C, 50.03; H, 3.48; N, 13.81 .

3-Bromo-1-(3-chloropyridin-2-yl)-N-(2-methyl-6-nitrophenyl)-1H-pyrazole-5-carboxamide (7k). Yield: 64.3\%. White solid, m.p. $143-145{ }^{\circ} \mathrm{C}$; ${ }^{1} \mathrm{H}-\mathrm{NMR}$ (DMSO- $\left.d 6,400 \mathrm{MHz}\right) \delta: 2.27\left(\mathrm{~s}, 3 \mathrm{H}, \mathrm{CH}_{3}\right), 7.39$ (s, 1H, pyrazolyl-H), 7.45 (t, 1H, $J=7.6 \mathrm{~Hz}, \mathrm{Ar}-\mathrm{H}), 7.61$ (dd, $J=4.8,8.0 \mathrm{~Hz}, 1 \mathrm{H}$, pyridyl-H), 7.65 (d, $1 \mathrm{H}, J=7.6 \mathrm{~Hz}, \mathrm{Ar}-\mathrm{H}), 7.79$ (d, $1 \mathrm{H}, J=7.6 \mathrm{~Hz}, \mathrm{Ar}-\mathrm{H}), 8.18$ (dd, $J=1.2,8.0 \mathrm{~Hz}, 1 \mathrm{H}$, pyridyl-H), 8.50 (dd, $1 \mathrm{H}, J=1.2,4.4 \mathrm{~Hz}, 1 \mathrm{H}$, pyridyl-H), 10.63 (br. s, $\mathrm{NH}$ ); Elemental anal. (\%), calcd. for $\mathrm{C}_{16} \mathrm{H}_{11} \mathrm{BrClN}_{5} \mathrm{O}_{3}$ : C, 44.01; H, 2.54; N, 16.04; found: C, 44.25; H, 2.69; N, 15.85 .

3-Bromo-N-(2-chloro-4-nitrophenyl)-1-(3-chloropyridin-2-yl)-1H-pyrazole-5-carboxamide (7l). Yield: $52.7 \%$. White solid, m.p. $176-17{ }^{\circ} \mathrm{C} ;{ }^{1} \mathrm{H}-\mathrm{NMR}\left(\mathrm{CDCl}_{3}, 400 \mathrm{MHz}\right) \delta: 6.99$ (s, 1H, pyrazolyl-H), 7.48 (dd, $J=4.8,8.4 \mathrm{~Hz}, 1 \mathrm{H}$, pyridyl-H), 7.96 (dd, $J=1.6,8.4 \mathrm{~Hz}, 1 \mathrm{H}$, pyridyl-H), 8.13 (dd, $1 \mathrm{H}, J=2.4$, 
$9.2 \mathrm{~Hz}, \mathrm{Ar}-\mathrm{H}), 8.34$ (d, 1H, $J=2.4 \mathrm{~Hz}, \mathrm{Ar}-\mathrm{H}), 8.51$ (dd, 1H, $J=1.2,4.4 \mathrm{~Hz}$, pyridyl-H), 8.55 (d, 1H, $J=9.2 \mathrm{~Hz}, \mathrm{Ar}-\mathrm{H}$ ), 8.62 (br. s, $1 \mathrm{H}, \mathrm{NH}$ ); Elemental anal. (\%), calcd. for $\mathrm{C}_{15} \mathrm{H}_{8} \mathrm{BrCl}_{2} \mathrm{~N}_{5} \mathrm{O}_{3}: \mathrm{C}, 39.42 ; \mathrm{H}$, 1.76 ; N, 15.32; found: C, 39.09; H, 2.01; N, 15.51 .

3-Bromo-N-(2-bromo-4-nitrophenyl)-1-(3-chloropyridin-2-yl)-1H-pyrazole-5-carboxamide (7m). Yield: 46.0\%. White solid, m.p. $176-178{ }^{\circ} \mathrm{C} ;{ }^{1} \mathrm{H}-\mathrm{NMR}\left(\mathrm{CDCl}_{3}, 400 \mathrm{MHz}\right) \delta: 6.99$ (s, 1H, pyrazolyl-H), 7.48 (dd, $J=4.8,8.4 \mathrm{~Hz}, 1 \mathrm{H}$, pyridyl-H), 7.96 (dd, $J=1.6,8.0 \mathrm{~Hz}, 1 \mathrm{H}$, pyridyl-H), 8.16 (dd, $1 \mathrm{H}, J=2.4$, 9.2 Hz, Ar-H), 8.49-8.53 (m, 1H, pyridyl-H, 2H, Ar-H), 8.60 (br. s, 1H, NH); Elemental anal. (\%), calcd. for $\mathrm{C}_{15} \mathrm{H}_{8} \mathrm{Br}_{2} \mathrm{ClN}_{5} \mathrm{O}_{3}$ : C, 35.92; $\mathrm{H}, 1.61 ; \mathrm{N}, 13.96$; found: $\mathrm{C}, 36.24 ; \mathrm{H}, 1.73 ; \mathrm{N}, 14.08$.

3-Bromo-N-(2-nitro-4-chlorophenyl)-1-(3-chloropyridin-2-yl)-1H-pyrazole-5-carboxamide (7n). Yield: 55.5\%. White solid, m.p. $193-194{ }^{\circ} \mathrm{C} ;{ }^{1} \mathrm{H}-\mathrm{NMR}\left(\mathrm{CDCl}_{3}, 400 \mathrm{MHz}\right) \delta: 7.02$ (s, 1H, pyrazolyl-H), 7.46 (dd, $J=4.8,8.0 \mathrm{~Hz}, 1 \mathrm{H}$, pyridyl-H), 7.57 (dd, 1H, $J=2.8,9.2 \mathrm{~Hz}, \mathrm{Ar}-\mathrm{H}), 7.94$ (dd, $J=1.2,8.0 \mathrm{~Hz}$, $1 \mathrm{H}$, pyridyl-H), 8.27 (d, 1H, $J=2.4 \mathrm{~Hz}, \mathrm{Ar}-\mathrm{H}), 8.51$ (dd, $1 \mathrm{H}, J=1.6,4.8 \mathrm{~Hz}$, pyridyl-H), $8.66(\mathrm{~d}, 1 \mathrm{H}$, $J=9.2 \mathrm{~Hz}, \mathrm{Ar}-\mathrm{H}$ ), 11.14 (br. s, $1 \mathrm{H}, \mathrm{NH}$ ); Elemental anal. (\%), calcd. for $\mathrm{C}_{15} \mathrm{H}_{8} \mathrm{BrCl}_{2} \mathrm{~N}_{5} \mathrm{O}_{3}$ : C, 39.42; $\mathrm{H}$, 1.76 ; N, 15.32; found: C, 39.76; H, 1.98; N, 15.80 .

3-Bromo-1-(3-chloropyridin-2-yl)-N-(2,5-dichlorophenyl)-1H-pyrazole-5-carboxamide (7o). Yield: 74.0\%. White solid, m.p. 190-192 ${ }^{\circ} \mathrm{C} ;{ }^{1} \mathrm{H}-\mathrm{NMR}\left(\mathrm{CDCl}_{3}, 400 \mathrm{MHz}\right) \delta: 6.94$ (s, 1H, pyrazolyl-H), 7.06-7.08 (m, 1H, Ar-H), 7.31-7.36 (m, 1H, Ar-H), 7.45-7.48 (m, 1H, pyridyl-H), $7.94(\mathrm{~d}, 1 \mathrm{H}, J=8.0 \mathrm{~Hz}$, pyridyl-H), 8.35 (s, $1 \mathrm{H}, \mathrm{NH}), 8.51(\mathrm{~d}, 1 \mathrm{H}, J=4.4 \mathrm{~Hz}$, pyridyl-H); Elemental anal. (\%), calcd. for $\mathrm{C}_{15} \mathrm{H}_{8} \mathrm{BrCl}_{3} \mathrm{~N}_{4} \mathrm{O}: \mathrm{C}, 40.35 ; \mathrm{H}, 1.81 ; \mathrm{N}, 12.55$; found: $\mathrm{C}, 40.28 ; \mathrm{H}, 2.06 ; \mathrm{N}, 12.32$.

3-Bromo-N-(4-chloro-2,6-dimethylphenyl)-1-(3-chloropyridin-2-yl)-1H-pyrazole-5-carboxamide (7p). Yield: 52.3\%. White solid, m.p. 225-228 ${ }^{\circ} \mathrm{C} ;{ }^{1} \mathrm{H}-\mathrm{NMR}\left(\mathrm{CDCl}_{3}, 400 \mathrm{MHz}\right) \delta: 2.14\left(\mathrm{~s}, 6 \mathrm{H}, \mathrm{CH}_{3}\right), 6.89$ (s, 1H, pyrazolyl-H), 7.04 (s, 2H, Ar-H), 7.38 (dd, $J=4.8,8.0 \mathrm{~Hz}, 1 \mathrm{H}$, pyridyl-H), 7.45 (br. s, 1H, $\mathrm{NH}), 7.87$ (dd, $1 \mathrm{H}, J=1.6,8.0 \mathrm{~Hz}$, pyridyl-H), 8.44 (dd, 1H, $J=1.6,4.4 \mathrm{~Hz}$, pyridyl-H); Elemental anal. (\%), calcd. for $\mathrm{C}_{17} \mathrm{H}_{13} \mathrm{BrCl}_{2} \mathrm{~N}_{4} \mathrm{O}: \mathrm{C}, 46.39 ; \mathrm{H}, 2.98 ; \mathrm{N}, 12.73$; found: $\mathrm{C}, 46.18 ; \mathrm{H}, 3.28 ; \mathrm{N}, 12.17$.

3-Bromo-N-(4-bromo-2,6-dimethylphenyl)-1-(3-chloropyridin-2-yl)-1H-pyrazole-5-carboxamide (7q). Yield: 55.2\%. White solid, m.p. 237-238 ${ }^{\circ} \mathrm{C} ;{ }^{1} \mathrm{H}-\mathrm{NMR}\left(\mathrm{CDCl}_{3}, 400 \mathrm{MHz}\right) \delta: 2.17\left(\mathrm{~s}, 6 \mathrm{H}, \mathrm{CH}_{3}\right), 6.92$ (s, 1H, pyrazolyl-H), 7.22 (s, 2H, Ar-H), 7.38 (dd, $J=4.8,8.0 \mathrm{~Hz}, 1 \mathrm{H}$, pyridyl-H), 7.41 (br. s, $1 \mathrm{H}$, $\mathrm{NH}), 7.86(\mathrm{dd}, 1 \mathrm{H}, J=1.6,8.0 \mathrm{~Hz}$, pyridyl-H), 8.45 (dd, 1H, $J=1.6,4.8 \mathrm{~Hz}$, pyridyl-H); Elemental anal. (\%), calcd. for $\mathrm{C}_{17} \mathrm{H}_{13} \mathrm{Br}_{2} \mathrm{ClN}_{4} \mathrm{O}: \mathrm{C}, 46.99 ; \mathrm{H}, 3.55 ; \mathrm{N}, 13.70$; found: $\mathrm{C}, 46.78 ; \mathrm{H}, 3.38 ; \mathrm{N}, 13.78$.

3-Bromo-N-(2-chloro-6-methyl-4-nitrophenyl)-1-(3-chloropyridin-2-yl)-1H-pyrazole-5-carboxamide (7r). Yield: 53.2\%. Yellow solid, m.p. $107-109{ }^{\circ} \mathrm{C}$; ${ }^{1} \mathrm{H}-\mathrm{NMR}$ (DMSO- $\left.d_{6}, 400 \mathrm{MHz}\right) \delta$ : $2.30\left(\mathrm{~s}, 3 \mathrm{H}, \mathrm{CH}_{3}\right.$ ), 7.45 (s, 1H, pyrazolyl-H), 7.62 (dd, $J=4.4,8.0 \mathrm{~Hz}, 1 \mathrm{H}$, pyridyl-H), 8.18-8.23 (m, 2H, pyridyl-H, Ar-H), 7.80-7.82 (m, 1H, Ar-H), 8.34 (d, 1H, $J=2.4 \mathrm{~Hz}, \operatorname{Ar}-\mathrm{H}), 8.51$ (dd, $1 \mathrm{H}, J=1.2,4.4 \mathrm{~Hz}$, pyridyl-H), 10.79 (br. s, NH); Elemental anal. (\%), calcd. for $\mathrm{C}_{16} \mathrm{H}_{10} \mathrm{BrCl}_{2} \mathrm{~N}_{5} \mathrm{O}_{3}$ : C, 40.79; H, 2.14; N, 14.87; found: C, 40.58; H, 2.21; N, 14.89 . 
3-Bromo-N-(4-chloro-2-methyl-6-nitrophenyl)-1-(3-chloropyridin-2-yl)-1H-pyrazole-5-carboxamide (7s). Yield: 46.6\%. White solid, m.p. $100-104{ }^{\circ} \mathrm{C} ;{ }^{1} \mathrm{H}-\mathrm{NMR}$ (DMSO- $\left.d_{6}, 400 \mathrm{MHz}\right) \delta: 2.28\left(\mathrm{~s}, 3 \mathrm{H}, \mathrm{CH}_{3}\right.$ ), 7.01 (s, 1H, pyrazolyl-H), 7.40 (dd, $J=4.8,8.4 \mathrm{~Hz}, 1 \mathrm{H}$, pyridyl-H), 7.51 (d, 1H, $J=2.4 \mathrm{~Hz}, \mathrm{Ar}-\mathrm{H})$, 7.87-7.90 (m, 2H, pyridyl-H, Ar-H), 8.47 (dd, 1H, $J=1.6,4.8 \mathrm{~Hz}$, pyridyl-H), 9.05 (br. s, 1H, NH); HRMS (ESI) $m / z$ : 491.9233 (Calcd for $\mathrm{C}_{16} \mathrm{H}_{10} \mathrm{BrCl}_{2} \mathrm{~N}_{5} \mathrm{O}_{3}[\mathrm{M}+\mathrm{Na}]^{+}$: 491.9236).

\subsection{Crystal Structure Determination}

The prism-shaped single crystal of the title compound was obtained by recrystallization from EtOH. The crystal with dimensions of $0.20 \mathrm{~mm} \times 0.16 \mathrm{~mm} \times 0.12 \mathrm{~mm}$ was mounted on a rigaku saturn diffractometer with a graphite-monochromated MoK $\alpha$ radiation $(\lambda=0.71073 \AA)$ by using a Phi scan modes at $113(2) \mathrm{K}$ in the range of $2.28 \leq \theta \leq 25.02$. The crystals are triclinic, space group P-1 with $a=8.8252(18), b=9.1389(18), c=10.448(2) \AA, \alpha=96.61(3), \beta=91.95(3), \gamma=99.49(3)^{\circ}, V=824.4(3)$ $\AA^{3}, Z=2, F(000)=452, D_{c}=1.841 \mathrm{~g} / \mathrm{cm}^{3}, \mu=0.285 \mathrm{~cm}^{-1}$. A total of 5604 reflections were collected, of which 2884 were independent $\left(R_{\text {int }}=0.0345\right)$ and 2197 were observed with $\mathrm{I}>2 \sigma(\mathrm{I})$. The calculations were performed with SHELXS-97 program [26] and the empirical absorption corrections were applied to all intensity data. The non-hydrogen atoms were refined anisotropically. The hydrogen atoms were determined with theoretical calculations and refined isotropically. The final full-matrix least squares refinement gave:

$$
\mathrm{R} 1=0.0345 \text { and } \mathrm{wR} 2=0.0785\left(w=1 /\left[\sigma^{2}\left(F_{o}{ }^{2}\right)+(0.0391 P)^{2}\right]\right.
$$

where $P=\left(F_{o}{ }^{2}+2 F_{c}{ }^{2}\right) / 3, \mathrm{~S}=1.07,(\Delta / \sigma)_{\max }=0.002, \Delta \rho_{\max }=0.46$ and $\Delta \rho_{\min }=-0.65 \mathrm{e} \AA^{-3}$.

Atomic scattering factors and anomalous dispersion corrections were taken from International Table for X-Ray Crystallography [27]. CCDC-893647 contains the supplementary crystallographic data for this paper. These data can be obtained free of charge at http://www.ccdc.cam.ac.uk/conts/retrieving.html or from the Cambridge Crystallographic Data Centre, 12 Union Road, Cambridge CB2 1EZ, UK; fax: +44-1223-336033; e-mail: deposit@ccdc.cam.ac.uk.

\subsection{Biological Assay}

All bioassays were performed on representative test organisms reared in the laboratory, which were repeated at $25 \pm 1{ }^{\circ} \mathrm{C}$ according to statistical requirements. Assessments were made on a dead/alive basis and evaluations are based on a percentage scale of $0-100$ in which $0=$ no activity and $100=$ total kill.

\subsubsection{Stomach Toxicity against Mythimna separata Walker}

The leaf dipping assay method was used for M. separata tests [28,29], in which the corn leaves were dipped into a test solution for $20 \mathrm{~s}$ and allowed to dry. The treated diet was placed into a $7 \mathrm{~cm}$ diameter Petri dish, and 10 fourth-instar M. separata larvae were released into the dish. The symptoms of affected larvae were observed at $24 \mathrm{~h}$ after the application, and percentage mortalities were evaluated $72 \mathrm{~h}$ after treatment. For comparative purposes, Rynaxypyr ${ }^{\mathrm{TM}}$ was tested under the same conditions. Each treatment was performed three times. 


\subsubsection{Toxicity against Culex pipiens pallens}

The immersion method assay was used for C. p. pallens tests [30], and concentrations of test compounds were adjusted by serial dilution of a stock solution of the compounds in acetone. Each compound in acetone was suspended in distilled water, 10 early fourth-instar larvae of C. p. pallens were put into glass cups $(125 \mathrm{~mL})$ containing each test solution $(100 \mathrm{~mL})$. Larvicidal activity was evaluated $72 \mathrm{~h}$ after treatment. For comparative purposes, Rynaxypyr ${ }^{\mathrm{TM}}$ was tested under the same conditions. Each treatment was performed three times.

\subsubsection{Stomach Toxicity against Plutella xylostella (Linnaeus, 1758) and Laphygma exigua Hübner}

The leaf dipping assay method was used for P. xylostella and L. exigua tests [31,32]. A stock solution of each test sample was prepared in dimethylformamide at a concentration of $200 \mathrm{mg} \mathrm{L}^{-1}$ and then diluted to the required concentration with water containing TW-20. Leaf disks $(6 \mathrm{~cm} \times 2 \mathrm{~cm})$ were cut from fresh cabbage leaves and then were dipped into the test solution for $3 \mathrm{~s}$. After air-drying, the treated leaf disks were placed individually into glass tubes. Each dried treated leaf disk was infested with seven second-instar P. xylostella larvae (third-instar L. exigua larvae). Percentage mortalities were evaluated three days after treatment. Leaves treated with water and dimethylformamide were provided as controls. For comparative purposes, Rynaxypyr ${ }^{\mathrm{TM}}$ was tested under the same conditions. Each treatment was performed three times.

\section{Conclusions}

In summary, a series of amides containing $N$-pyridylpyrazoles were synthesized and assessed for their insecticidal activities in vivo, using Rynaxypyr ${ }^{\mathrm{TM}}$ as reference control. Several of the synthesized compounds exhibited significant insecticidal activity on a broad spectrum of Lepidoptera. Compared with the anthranilic diamide insecticide Rynaxypyr ${ }^{\mathrm{TM}}$, the removal of the aliphatic amide moiety $(\mathbf{Z})$ from the anthraniloyl skeleton resulted in slightly decreased insecticidal efficacy. This implies that the aliphatic amide moiety might not be the insecticidal pharmacophore. The present findings provided a powerful complement to the SARs of amide insecticides, and warrant future investigation of the mechanism of action of these analogues.

\section{Acknowledgments}

This work was supported financially by the National Natural Science Funds of China (grant No. 21102103), the China Postdoctoral Science Foundation (grant No. 2011M500532).

\section{Conflict of Interest}

The authors declare no conflict of interest.

\section{References}

1. Nauen, R. Insecticide mode of action: Return of the ryanodine receptor. Pest Manag. Sci. 2006, 62, 690-692. 
2. Lahm, G.P.; Pasteris, R.J.; Stevenson, T.M. Pyrazole and pyrrole carboxamide insecticides. WO Patent 2,003,106,427, 24 December 2003; Chem. Abstr. 2003, 140, 42172.

3. Ebbinghaus-Kintscher, U.; Luemmena, P.; Lobitz, N.; Schulte, T.; Funke, C.; Fischer, R.; Masaki, T.; Yasokawa, N.; Tohnishi, M. Phthalic acid diamides activate ryanodine-sensitive $\mathrm{Ca}^{2+}$ release channels in insects. Cell Calcium 2006, 39, 21-33.

4. Cordova, D.; Benner, E.A.; Sacher, M.D.; Rauh, J.J.; Sopa, J.S.; Lahm, G.P.; Selby, T.P.; Stevenson, T.M.; Flexner, L.; Gutteridge, S.; et al. Anthranilic diamides: A new class of insecticides with a novel mode of action, Ryanodine receptor activation. Pest. Biochem. Physiol. 2006, 84, 196-214.

5. Lahm, G.P.; Selby, T.P.; Freudenberger, J.H.; Stevenson, T.M.; Myers, B.J.; Seburyamo, G.S.; Smith, B.K.; Flex-ner, L.; Clark, C.E.; Cordova, D. Insecticidal anthranilic diamides: A new class of potent ryanodine receptor activators. Bioorg. Med. Chem. Lett. 2005, 15, 4898-4906.

6. Lahm, G.P.; Stevenson, T.M.; Selby, T.P.; Freudenberger, J.H.; Cordova, D.; Flexner, L.; Bellin, C.A.; Dubas, C.M.; Smith, B.K.; Hughes, K.A.; et al. Rynaxypyr (TM): A new insecticidal anthranilic diamide that acts as a potent and selective ryanodine receptor activator. Bioorg. Med. Chem. Lett. 2007, 17, 6274-6279.

7. Dong, W.L.; Xu, J.Y.; Liu, X.H.; Li, Z.M.; Li, B.J.; Shi, Y.X. Synthesis, Crystal structure and biological activity of novel anthranilic diamides containing 1,2,3-thiadiazole. Chem. J. Chin. Univ. 2008, 29, 1990-1994.

8. Dong, W.L.; Liu, X.H.; Xu, J.Y.; Li, Z.M. Design and synthesis of novel anthranilic diamides containing 5,7-dimethyl[1,2,4]triazolo[1,5-a]pyrimidine. J. Chem. Res. 2008, 530-533.

9. Dong, W.L.; Xu, J.Y.; Liu, X.H.; Xiong, L.X.; Li, Z.M. Synthesis, Structure and biological activities of some novel anthranilic acid esters containing $N$-Pyridylpyrazole. Chin. J. Chem. 2009, 27, 579-586.

10. Xu, J.Y.; Dong, W.L.; Xiong, L.X.; Li, Y.X.; Li, Z.M. Design, Synthesis and biological activities of novel amides (Sulfonamides) containing $N$-Pyridylpyrazole. Chin. J. Chem. 2009, 27, 2007-2012.

11. Shapiro, R.; Taylor, E.G.; Zimmerman, W.T. Method for preparing N-phenylpyrazole-1carboxamides. WO Patent 2,006,062,978, 15 June 2006; Chem. Abstr. 2006, 145, 62887.

12. Lahm, G.P.; Selby, T.P.; Stevenson, T.M. Arthropodicidal anthranilamides. WO Patent 2,003,015,519, 27 February 2003; Chem. Abstr. 2003, 138, 200332.

13. Liu, X.H.; Pan, L.; Tan, C.X.; Weng, J.Q.; Wang, B.L.; Li, Z.M. Synthesis, Crystal structure, Bioactivity and DFT calculation of new oxime ester derivatives containing cyclopropane moiety. Pestic. Biochem.Physiol. 2011, 101, 143-147.

14. Xue, Y.L.; Zhang, Y.G.; Liu, X.H. Synthesis, Crystal structure and biological activity of 1-CyanoN-(4-bromophenyl)cyclopropanecarboxamide. Asian J. Chem. 2012, 24, 3016-3018.

15. Liu, X.H.; Pan, L.; Weng, J.Q.; Tan, C.X.; Li, Y.H.; Wang, B.L.; Li, Z.M. Synthesis, Structure, and biological activity of novel (oxdi/tri)azoles derivatives containing 1,2,3-thiadiazole or methyl moiety. Mol. Divers. 2012, 16, 251-260.

16. Tan, C.X.; Weng, J.Q.; Liu, Z.X.; Liu, X.H.; Zhao, W.G. Synthesis, Crystal structure, and Fungicidal activity of a novel 1,2,3-Thidiazole compound. Phosphorus Sulfur Silicon Relat. Elem. 2012, 187, 990-996. 
17. Liu, X.H.; Tan, C.X.; Weng, J.Q.; Liu, H.J. (E)-(4-Bromobenzylidene)amino cyclopropanecarboxylate. Acta Cryst. 2012, 68, o493.

18. Liu, X.H.; Tan, C.X.; Weng, J.Q. Synthesis, Dimeric crystal structure, and Fungicidal activity of 1-(4-Methylphenyl)-2-(5-((3,5-Dimethyl-1H-Pyrazol-1-yl)methyl)-4-Phenyl-4H-1,2,4-Trizol3-ylthio)Ethanone. Phosphorus Sulfur Silicon Relat. Elem. 2011, 186, 558-564.

19. Liu, X.F.; Liu, X.H. 5-(4-Pyridyl)-1,3,4-thiadiazole-2(3H)-thione. Acta Cryst. 2011, 67, o202.

20. Chen, P.Q.; Tan, C.X.; Weng, J.Q.; Liu, X.H. Synthesis, Structure and DFT calculation of chlorimuron-ethyl. Asian J. Chem. 2012, 24, 2808-2810.

21. Xue, Y.L.; Liu, X.H.; Zhang, Y.G. Synthesis, Crystal structure and biological activity of 1-CyanoN-phenylcyclopropanecarboxamide. Asian J. Chem. 2012, 24, 1571-1574.

22. Liu, H.J.; Weng, J.Q.; Tan, C.X.; Liu, X.H. 1-Cyano-N-(2,4,5-trichlorophenyl)cyclopropane-1carboxamide. Acta Cryst. 2011, 67, o1940.

23. Xue, Y.L.; Zhang, Y.G.; Liu, X.H. Synthesis, Crystal structure and biological activity of 1-Cyano$\mathrm{N}$-(2,4-dichlorophenyl)cyclopropanecarboxamide. Asian J. Chem. 2012, 24, 5087-5089.

24. Liu, X.H.; Pan, L.; Ma, Y.; Weng, J.Q.; Tan, C.X.; Li, Y.H.; Shi, Y.X.; Li, B.J.; Li, Z.M.; Zhang, Y.G. Design, Synthesis, Biological activities, and 3D-QSAR of new N,N'-Diacylhydrazines containing 2-(2,4-dichlorophenoxy)propane Moiety. Chem. Biol. Drug Des. 2011, 78, 689-694.

25. Clark, D.A.; Lahm, G.P.; Smith, B.K.; Barry, J.D.; Clagg, D.G. Synthesis of insecticidal fluorinated anthranilic diamides. Bioorg. Med. Chem. 2008, 16, 3163-3170.

26. Sheldrick, G.M. SHELXS97 and SHELXL97; University of Göttingen: Göttingen, Germany, 1997.

27. Wilson, A.J. International Table for X-ray Crystallography; Kluwer Academic Publisher: Dordrecht, The Netherlands, 1992; Volume C, pp. 219-222, 500-502.

28. Abbott, W.S. A method of computing the effectiveness of an insecticide. J. Econ. Entomol. 1925, $18,265-267$.

29. Zhao, Q.Q.; Li, Y.Q.; Xiong, L.X.; Wang, Q.M. Design, Synthesis and insecticidal activity of novel phenylpyrazoles containing a 2,2,2-Trichloro-1-alkoxyethyl moiety. J. Agric. Food Chem. 2010, 58, 4992-4998.

30. Raymond, M.; Marquine, M. Evolution of insecticide resistance in Culex pipiens polulations: The Corsican paradox. J. Evol. Biol. 1994, 7, 315-337.

31. Sun, R.F.; Zhang, Y.L.; Chen, L.; Li, Y.Q.; Li, Q.S.; Song, H.B.; Huang, R.Q.; Bi, F.C.; Wang, Q.M. Design, Synthesis and insecticidal activities of new $N$-Benzoyl- $N^{\prime}$-phenyl- $N^{\prime}$-sulfenylureas. J. Agric. Food Chem. 2009, 57, 3661-3668.

32. Sayyed, A.H.; Ferre, J.; Wright, D.J. Mode of inheritance and stability of resistance to Bacillus thuringiensis var kurstaki in a diamondback moth (Plutella xylostella) population from Malaysia. Pest Manage. Sci. 2000, 56, 743-748.

Sample Availability: Samples of the compounds are available from the authors.

(C) 2012 by the authors; licensee MDPI, Basel, Switzerland. This article is an open access article distributed under the terms and conditions of the Creative Commons Attribution license (http://creativecommons.org/licenses/by/3.0/). 